\title{
Quench Observation Using Quench Antennas on RHIC IR Quadrupole Magnets
} CONF- $950691--13$

\author{
T. Ogitsu, A. Terashima, and K. Tsuchiya \\ KEK, National Laboratory for High Energy Physics, Tsukuba, Ibaraki, Japan \\ G. Ganetis, J. Muratore, and P. Wanderer \\ Brookhavea Nacional Laboratory, Upton, NY 11973 USA
}

\begin{abstract}
Quench observation using quench antennas is now being performed routinely on RHIC dipole and quadrupole magnets. Recently, a quench antenna was used on a RHIC IR magaet which is hearily instrumented with voltage taps. It was confrmed that the signals detected in the antenna colls do not contradict the voltage tap signals. The antenna also detects a sign of mechanical disturbance which could be related to a training quench. This paper surmarizes signals detected in the antenna and discusses possible causes of these slgnals.
\end{abstract}

\section{INTRODUCTION}

Tests of superconducting magnets for the Relativistic Heavy Ion Collider (RHIC) project are now being performed at Brookhaven National Laboratory (BNL). Most of these magnets are not instrumented with voltage taps which enable us to localize a quench origin [1]. To localize quenches in these production magnets, several quench antennas bave been developed jointly by KEK and BNL [2]. These antennas are based on the antennas developed for SSC dipole magnets [3] which were evolved from the method developed at CERN [4]. The method developed at CERN, named quench localization coils (QLC) [5], uses sets of pickup coils which detect field fluctuations introduced by quenches. The quench antennas rely on the same concept but use sets of pickup coils which are only sensitive to higher order multipole fields. This configuration eliminates the need to detect fluctuations of the main multipole field which contains the noise of the power supply, but is still sensitive to fluctuations caused by quenches. Recently, the antenna for the Interaction Region (IR) quadrupole magnets [6] has been used with magnets which are heavily instrumented with voltage taps [2]. It was confirmed that the signals detected in the antenna coils do not contradict the voltage tap signals. The antenna also detects indications of mechanical disturbances in training quenches. This paper summarizes examples of the antenna signals and discusses origins of these signals.

Manuscript reccived June 12th 1995.

T.Ogitsu, e-mail ogitsu@kekvax.kek.jp, fax +81-298-64-3182, A.Terasima, fax +81-298-64-5590, K.Tsuchiyz, e-mail kiyosumiekekrax.kek.jp, fax +81-298-64-3182, G.Ganetis, e-mail BNLADO::GANETIS, J.Muratore, email BNLADO::MURATORE, P.Wanderer, c-mail wandererebal.gov Fax $+1-516-282-2190$

This work was supported in part by the U.SJJapan Collaboration in the Field of High Energy Physics.

\section{SYSTEM CONFIGURATION}

The antenna used for the tests introduced here consists of two coil arrays which are lined up along the magnet length. Each coil array contains four pickup coils; the first one is sensitive to normal sextupole, the second to skew sextupole, the third to normal octupole, and the fourth to skew octupole field. Fig. la presents a cross-sectional view of a coil array. A three dimensional view of a single turn sextupole coil is shown in Fig. 1b. It was shown by Morgan [7] that this kind of winding is primarily sensitive to the sextupole field. The octupole coil is similar to the sextupole except that the wire crosses the coil form every $\pi / 4$. The skew coils are rotated from the normal coils by $\pi / 6$ for sextupole and $\pi / 8$ for octupole coils.

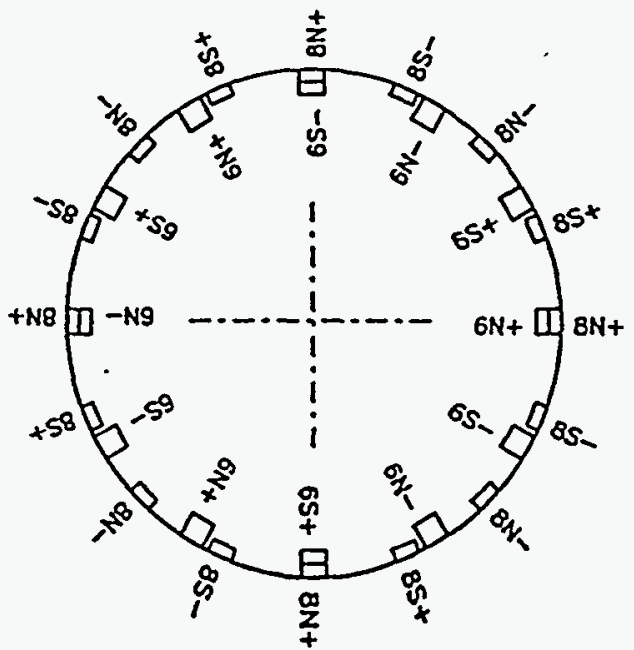

Fig. ta. Cross-sectional view of a coil array. 6N, 6S, 8N, and 85 indicate normal sextupole, skew sextupole, normal octupole, and skew octupole. respectively.

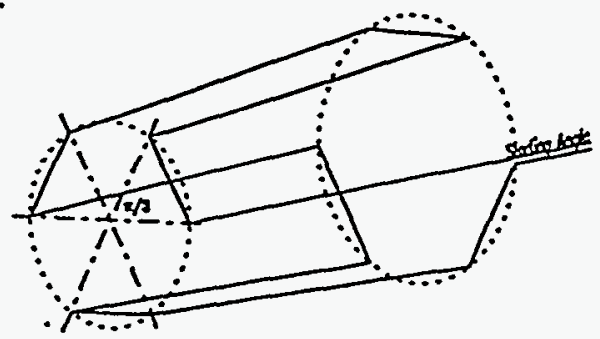

Fig. 1b. Three dimensional view of a single turn sextupole coil.

The length of cach coil is $150 \mathrm{~mm}$, and the diameter is $79 \mathrm{~mm}$. The centers of the two coil arrays are located 155 
$\mathrm{min}$ from the center of the magnet toward the ends. The coil array close to the lead end is named C-1 and the other is C-2.

The measurements were made on the magnet named QRI999, which is a $1.3 \mathrm{~m}$ long, $13 \mathrm{~cm}$ aperture model IR quadrupole magnet. Single layer, $\cos (2 \theta)$ coils produce a nominal field gradient of $48.1 \mathrm{~T} / \mathrm{m}$ at the operating current of $5.0 \mathrm{kA}$. The coils are wound from a Rutherford-type cable which consists of 36 strands and has mid-thickness 1.156 $\mathrm{mm}$, cable width $11.68 \mathrm{~mm}$, and cable lay pitch $94 \mathrm{~mm}$. Detailed features of the magnet can be seen in [6].

The antenna signals and the voltage tap signals are taken using the same data acquisition system with sampling rate 5 $\mathrm{kHz}$. The system is triggered when the amplitude of the balance signal, the voltage difference between coil 1-2 and 34 , exceeds a threshold of $2 \mathrm{~V}$.

\section{MEASUREMENTS}

Examples of measured signals presented here are taken during the first testing cycle. Quench currents of this cycle are summarized in Fig. 2. All the quenches are performed at a ramp rate of $16 \mathrm{~A} / \mathrm{s}$. Up to the third quench, the current is ramped in $500 \mathrm{~A}$ steps until a quench occurs. Between the second and third quench, 10 current cycles to $7 \mathrm{kA}$ and a 1 hour hold at $7 \mathrm{kA}$ were performed. For the remaining quenches, the current is ramped to quench without stopping.

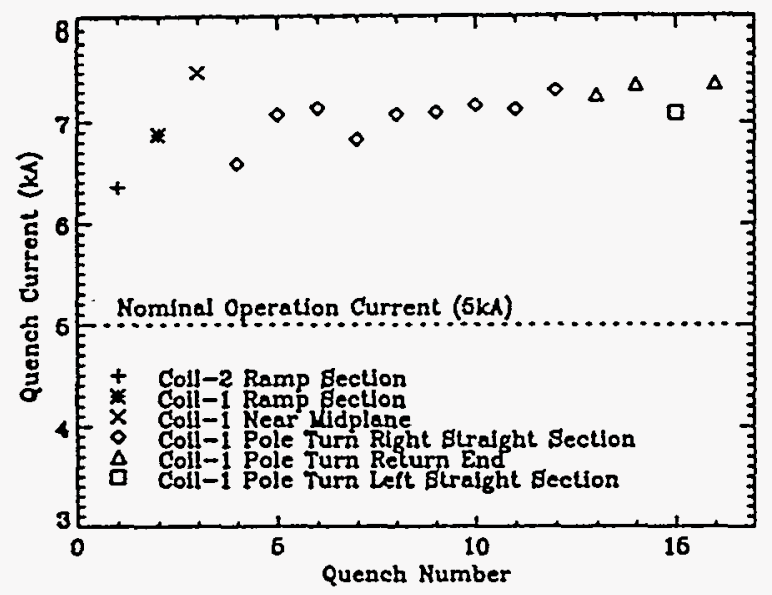

Fig. 2. Quench summary of the first testing cycle of magnet QRI999. Different plot symbols indicate different quench start locations. The locations are derived from the voltage tap signals. Numbers of the coils count clockwise starting from the upper left coil as viewed from the magnet lead end.

\section{A. Quench with Mechanical Disturbance}

Fig. 3 presents signals of the antenna coils in array C-1 and the balance signal taken during the first spontaneous quench. The quench current is $6348 \mathrm{~A}$, and the voltage tap signals indicate that the quench origin is at the ramp splice section in coil 2 of the magnet. The balance signal shows a voltage spike which suggests the existence of a mechanical disturbance [8]. The signals of the antenna coils show a spike associated with oscillation of the signal which can be related to this mechanical disturbance [9]. The oscillation is larger in the signals of the octupole coils than that of sextupole coils indicating that the main component of this field fluctuation is octupole field. The octupole field fluctuation can be introduced by a mechanical vibration which produces an elliptical deformation of the coil cross-section. A distortion where the axes of the ellipse are at the midplanes of coils introduces normal octupole while the other, i.e. the axes of the ellipse at the poles, causes skew octupole field. The amplitude of the oscillation seen in the signals is about 20 $\mu \mathrm{V}$ and the frequency is about $1 \mathrm{kHz}$. This corresponds to a mechanical vibration whose maximum distortion in the inner diameter of the magnet coil is about $0.2 \mu \mathrm{m}$. This kind of oscillation can be seen in the first two quenches of the first thermal cycle. As described later, the oscillation has not been observed in the other quenches of this magnet.

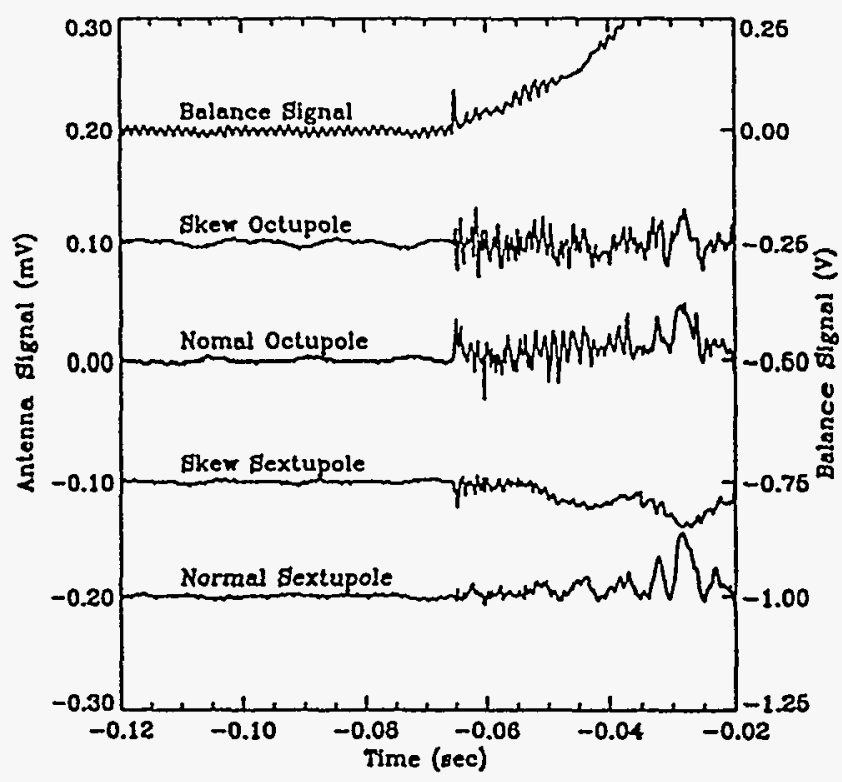

Fig. 3. Antenna coil signals taken during the first quench of the first testing cycle.

\section{B. Quenches at Various Quench Currents}

After two training quenches, the third quench reached $7505 \mathrm{~A}$, the highest quench current in this testing cycle. After this quench, the quench current fluctuated between $6589 \mathrm{~A}$ and $7276 \mathrm{~A}$ for the next 9 quenches, though the origins of these quenches were all in the same location. Fig. 4 presents signals of the antenna coils in array $\mathrm{C}-1$ and the balance signal taken during the sixth quench. The quench current is $7140 \mathrm{~A}$, and the quench start location is at the straight section of the left pole turn in coil 1. All the signals of the antenna coils of array $\mathrm{C}-1$ and the balance signal begin almost simultaneously indicating that the axial location of the quench origin is within the axial region covered by coil array C-1. This agrees with the quench localization based on the 


\section{DISCLAIMER}

This report was prepared as an account of work sponsored by an agency of the United States Government. Neither the United States Government nor any agency thereof, nor any of their employees, make any warranty, express or implied, or assumes any legal liability or responsibility for the accuracy, completeness, or usefulness of any information, apparatus, product, or process disclosed, or represents that its use would not infringe privately owned rights. Reference herein to any specific commercial product, process, or service by trade name, trademark, manufacturer, or otherwise does not necessarily constitute or imply its endorsement, recommendation, or favoring by the United States Government or any agency thereof. The views and opinions of authors expressed herein do not necessarily state or reflect those of the United States Government or any agency thereof. 


\section{DISCLAIMER}

Portions of this document may be illegible in electronic image products. Images are produced from the best available original document. 
volltage tap signals. Unlike the case of the training quenches these signals do not show oscillations similar to those seen in Fig. 3. This is true for the other eight quenches in this group. Fig. 5 presents signals of the normal octupole coil in array $\mathrm{C}-1$ for the quenches at $6589,6832,7071,7140$, and 7325 A. None of these signals shows oscillations similar to that seen in the training quenches. Although mechanical disturbances can not be totally eliminated as a cause of the erratic fluctuation of the quench currents, the antenna signals do not show an indication of a mechanical disturbance.

Another observation which can be clearly made is that

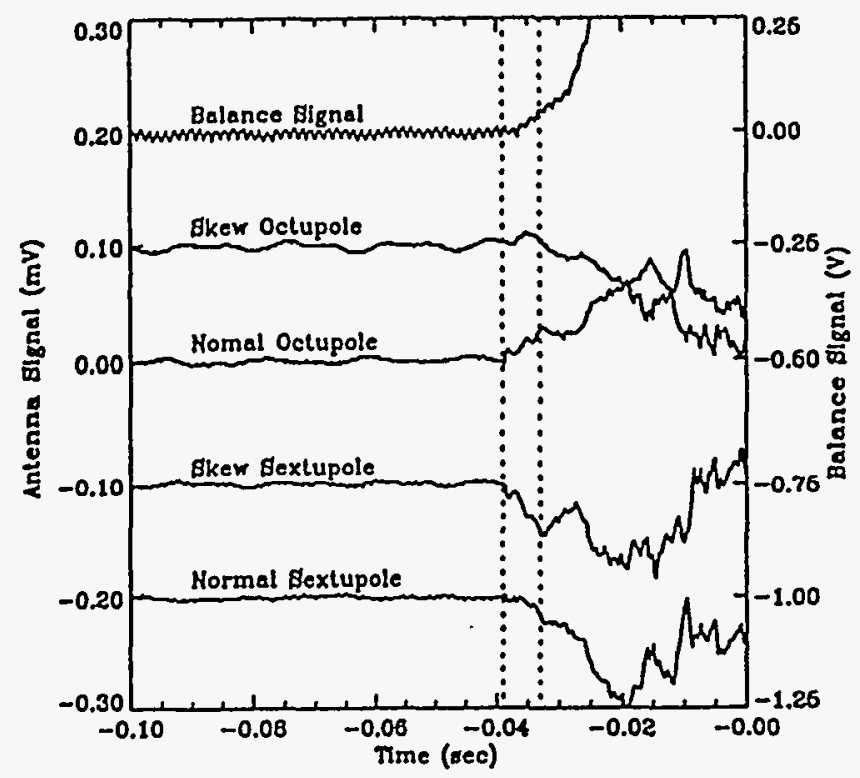

Fig. 4. Antenna coil signals taken during the sixth quench of the first testing cycle.

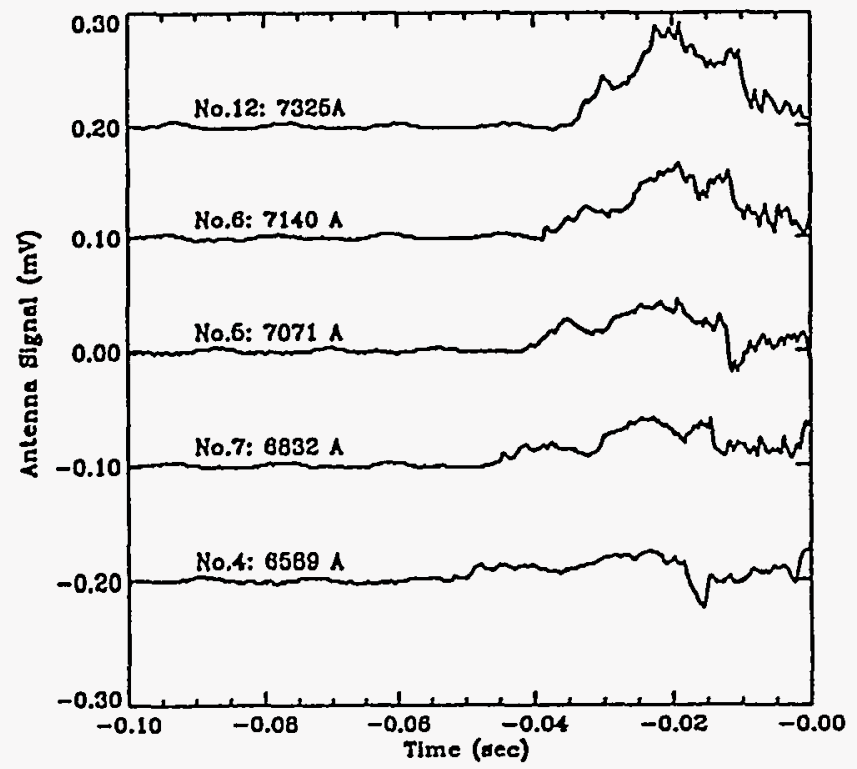

Fig. 5. Normal octupole coil signals taken during the quenches of the first testing cycle. Indicated are the quench numbers and the quench currents . the amplitude of the signals increases as the quench current increases. The shapes of the signals, however, are roughly similar to each other. These observations can be made for all the antenna coils. Similar signal shapes indicate that the current redistribution, which causes the field fluctuation, proceeds in a similar way in these quenches. Although the causes of the amplitude increase are not clear, it is useful to consider two reasons: 1) larger field gradient on the cable, and 2) faster quench velocity.

A field fluctuation which produces antenna signals is considered to be caused by current redistribution at the quench front. The current redistribution may be introduced by a field gradient across the cable cross-section. The quench front may proceed faster at points where the field is higher. At the quench front, therefore, current may redistribute from the high field region, where the conductor transits to the normal conducting state, to the low field region of the cable, where the conductor remains superconducting. Another mechanism which can be considered is that, at the normal conducting state, the conductor has higher resistance at the higher field region because of the magneto-resistance. This may result in a low current density in the high field region. In the case of the superconducting state, however, the average current density over one twist pitch length should be constant across the cable cross-section. The difference in the current density, therefore, should cause a current redistribution in the super-tonormal transition. Both mechanisms may be enhanced if the field gradient is larger. Since the field distribution in the magnet cross-section is approximately proportional to the magnet current, when the magnet current is higher the field gradient across a cable cross-section will be larger. These assumptions are reinforced by the fact that both quench signal amplitude and the field gradient are higher at the midplane than at the pole.

The above mechanisms define the amount of current redistribution during the super-to-normal transition. The amplitude of the signal, however, can be influenced by the other factor which is speed of the current redistribution. Quench velocity may be a dominant factor which decides the speed of the redistribution. In the case of the low current quenches, the quench velocities are slower than those of the higher current quenches. This results in a larger amplitude of the signals for the higher current quenches. The fact that the signals for the lower current quenches seem to be stretched over a longer time interval also indicates a slower transition.

\section{Two Dimensional Model}

Following the analysis performed in [2], we assume that the field distortion is produced by a moving current line. Let us define a Cartesian coordinate system such that the z-axis is parallel to the coil array and the origin is at the center of the coil array. The current $I_{s}$ parallel to the $z$-axis positioned at $(x, y)=r_{s} e^{i \alpha}$, moving with velocity $\left(v_{x}, v_{y}\right)=v_{s} e^{i \beta}$, induces voltages approximated as, 


$$
\begin{aligned}
& V_{6 n}=N_{6 n} \frac{3 r_{6 n}{ }^{3} \mu_{0} L_{6 n} I_{s} v_{s}}{\pi r_{s}{ }^{4}} \cos (-4 \alpha+\beta) \\
& V_{6 s}=N_{6 s} \frac{3 r_{6 s}{ }^{3} \mu_{0} L_{6 s} I_{s} v_{s}}{\pi r_{s}{ }^{4}} \sin (-4 \alpha+\beta) \\
& V_{8 n}=N_{8 n} \frac{4 r_{8 n} \mu_{0} \mu_{8 n} L_{s} v_{s}}{\pi r_{s}{ }^{5}} \cos (-5 \alpha+\beta) \\
& V_{8 s}=N_{8 s} \frac{4 r_{8 s}{ }^{4} \mu_{0} L_{8 s} I_{s} v_{s}}{\pi r_{s}{ }^{5}} \sin (-5 \alpha+\beta)
\end{aligned}
$$

where the subscripts $6 n, 6 s, 8 n$, and $8 s$ used for the voltage $V$, the number of turns $N$, the length $L$ and the radius $R$, refer to the normal sextupole, skew sextupole, normal octupole, and skew octupole coil, respectively. The system of equations allows us to obtain four unknowns, $r_{s}, \alpha, I_{s} v_{s}$ and $\beta$, analytically. Fig. 6 summarizes these four values derived for the time range indicated by the pair of dotted vertical lines in Fig. 4. The beginning of the range is the time when the signals start to rise. The end of the range is the time when the normal -zone appears to occupy the axial region covered by coil array $\mathrm{C}-\mathrm{l}$. The diamonds present the position given by $\mathrm{r}_{s}$ and $\alpha$ and the length and direction of the lines indicate $I_{s} v_{s}$ and $\beta$, respectively. One diamond represents each sampling step where the sampling rate is $5 \mathrm{kHz}$. A unit length of the line, i.c. $1 \mathrm{~m}$, corresponds to a current movement of 2000 $A \cdot m / s e c$. The diamonds are mostly located around the right pole turn of coil 1 . The voltage tap signals show that the quench starts in the straight section of the right pole turn in coil 1. Although the radial localizations from the antenna signals are slightly off from the cable, the azimuthal localizations are in good agreement with that from the voltage taps. The maximum amplitude of the current movement is about $100 \mathrm{~A} \cdot \mathrm{m} / \mathrm{sec}$, and its direction is roughly perpendicular to a flux line which crosses the pole turn. This indicates that the current moves from the high field region to the low field region in the cable cross-section. The integrated amplitude over the range is about $0.2 \mathrm{~A} \cdot \mathrm{m}$. This corresponds to a current redistribution of about $300 \mathrm{~A}$, assuming that the current moves along the cable thickness.

A current redistribution during the super-to-normal

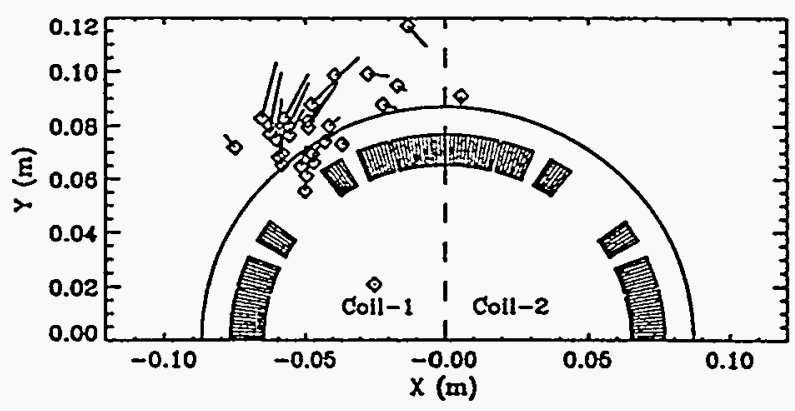

Fig. 6 Azimuthal quench localization, as viewed from the lead end. transition can be estimated from the properties of the conductor by assuming that the main cause is the magnetoresistance of the matrix copper in the cable. Assuming a residual resistivity ratio of 100 for the matrix copper, the current redistribution in the pole turn along the cable thickness is estimated to be about $700 \mathrm{~A}$. This estimation shows that the current redistribution of $300 \mathrm{~A}$, which is derived from the antenna signals, is a reasonable value.

\section{CONCLUSION}

Quench analyses using the quench antenna have been performed on a RHIC IR quadrupole magnet. The analyses indicate the existence of mechanical disturbances during the first two quenches. It was confirmed that the antenna detect the field fluctuation produced at a quench front. The analysis of the signals based on the crude two dimensional model appears to give good azimuthal localization of the quench front. It is, nevertheless, desirable to develop more sophisticated models in order to understand the exact nature of the antenna signals.

\section{ACKNOWLEDGMENT}

The authors would like to thank the members of the magnet test group of the RHIC project at BNL for their help. The authors would like to thank Prof. S. Ozaki for his kind support.

\section{REFERENCES}

[1] A. Devred, et al, "Quench Start Localization in Full-Length SSC R\&D Dipoles," Supercollider-1, M. McAshan ed., Plenum Press, N.Y.. 1989. p.73.

[2] T. Ogitsu, et al., "Quench Antennas for RHIC Quadrupole Magnets," presented at Particle Accelerator Conf., Dallas, TX, USA, May 1-5, 1995.

[3] T. Ogitsu, et al., "Quench Antenna for Superconducting Particle Accelerator Magnets," IEEE Trans. on Mag., Vol.30 No.4, 1994, p.2273.

[4] D. Leroy, et al., "Quench Observation in LHC Superconducting One Meter Long Dipole Model by Field Perturbation Mesurements," IEEE Trans. on Applied Superconductivity, Vol.3 No.1, 1993, p.781.

[5] A. Siemko, et al., "Quench Localization in the Superconducting Model Magnets for the LHC by Means of Pick-up Coils," presented at Applied Superconductivity Conf., Boston, MA, USA, 1994.

[6] G. Gupta, et al., "Large Aperture Quadrupoles for RHIC Interaction Regions," Proc. of 1993 Particle Accelerator Conf., vol. 4, p. 2745.

[7] G. Morgan "Stationary Coil for Measuring the Harmonics in Pulsed Transport Magnets," Proc. of 4th Int. Conf. on Mag. Tech., BNL, Upton NY, USA, Sep. 19-22, 1972, p. 787.

[8] T. Ogitsu, K. Tsuchiya and A. Devred, "Investigation of Wire Motion in Superconducting Magnets," IEEE Trans. on Mag., Vol.27, No.2, (March 1991), p.1743

[9] A.F. Lietzke, et al., "Quench Antenna \& Fast Motion Investigation During Training of a TT Dipole," presented at Applied Superconductivity Conf., Boston, MA, USA, 1994. 